\title{
TRIPLE AND QUADRUPLE JUNCTIONS THERMOPHOTOVOLTAIC DEVICES LATTICE MATCHED TO InP.
}

\author{
L. Bhusal \\ A. Freundlich \\ Photovolatics and Nanostructures Laboratories, Center for Advanced Materials and Physics Department, \\ University of Houston, Houston, TX-77204, USA
}

Themophotovoltaic (TPV) conversion of IR radiation emanating from a radioisotope heat source is under consideration for deep space exploration. Ideally, for radiator temperatures of interest, the TPV cell must convert efficiently photons in the $0.4-0.7 \mathrm{eV}$ spectral range. Best experimental data for single junction cells are obtained for lattice-mismatched $0.55 \mathrm{eV}$ InGaAs based devices. It was suggested, that a tandem InGaAs based TPV cell made by monolithically combining two or more lattice mismatched InGaAs subcells on InP would result in a sizeable efficiency improvement. However, from a practical standpoint the implementation of more than two subcells with lattice mismatch systems will require extremely thick graded layers (defect filtering systems) to accommodate the lattice mismatch between the sub-cells and could detrimentally affect the recycling of the unused IR energy to the emitter. A buffer structure, consisting of various InPAs layers, is incorporated to accommodate the lattice mismatch between the high and low bandgap subcells. There are evidences that the presence of the buffer structure may generate defects, which could extend down to the underlying InGaAs layer.

The unusual large band gap lowering observed in $\mathrm{GaAs}_{1-x} \mathrm{~N}_{\mathrm{x}}$ with low nitrogen fraction [1] has sparked a new interest in the development of dilute nitrogen containing III-V semiconductors for long-wavelength optoelectronic devices (e.g. IR lasers, detector, solar cells) [2-7]. Lattice matched $\mathrm{Ga}_{1-y} \mathrm{In}_{\mathrm{y}} \mathrm{N}_{\mathrm{x}} A \mathrm{~s}_{1-\mathrm{x}}$ on $\operatorname{InP}$ has recently been investigated for the potential use in the mid-infrared device applications [8], and it could be a strong candidate for the applications in TPV devices. This novel quaternary alloy allows the tuning of the band gap from $1.42 \mathrm{eV}$ to below $1 \mathrm{eV}$ on $\mathrm{GaAs}$ and band gap as low as $0.6 \mathrm{eV}$ when strained to $\ln P$, but it has its own limitations. To achieve such a low band gap using the quaternary $\mathrm{Ga}_{1-y} I \mathrm{n}_{\mathrm{y}} \mathrm{N}_{\mathrm{x}} \mathrm{As} \mathrm{s}_{1-\mathrm{x}}$, either it needs to be strained on $\operatorname{InP}$, which creates further complications due to the creation of defects and short life of the device or to introduce high content of indium, which again is found problematic due to the difficulties in diluting nitrogen in the presence of high indium [9]. An availability of material of proper band gap and lattice matching on InP are important issues for the development of TPV devices to perform better. To address those issues, recently we have shown that by adjusting the thickness of individual sublayers and the nitrogen composition, strain balanced $\mathrm{GaAs}_{1-\mathrm{x}} \mathrm{N}_{\mathrm{x}} / \mathrm{In} A \mathrm{~s}_{1-\mathrm{y}} \mathrm{N}_{\mathrm{y}}$ superlattice can be designed to be both lattice matched to $\ln P$ and have an effective bandgap in the desirable 0.4$0.7 \mathrm{eV}$ range $[10,11]$. Theoretically the already reduced band gap of $\mathrm{GaAs}_{1-\mathrm{x}} \mathrm{N}_{\mathrm{x}}$, due to the nitrogen effects, can be further reduced by subjecting it to a biaxial tensile strain, for example, by fabricating pseudomorphically strained layers on commonly available InP substrates. While such an approach in principle could allow access to smaller band gap (longer wavelength), only a few atomic monolayers of the material can be grown due to the large lattice mismatch between $\mathrm{GaAs}_{1-\mathrm{x}} \mathrm{N}_{\mathrm{x}}$ and $\operatorname{InP}(\sim 3.8-4.8 \%$ for $x<0.05,300 \mathrm{~K})$. This limitation can be avoided using the principle of strain balancing [12], by introducing the alternating layers of $\ln A s_{1-y} N_{y}$ with opposite strain $(\sim 2.4-3.1 \%$ for $x<0.05,300 K)$ in combination with $\mathrm{GaAs}_{1-\mathrm{x}} \mathrm{N}_{\mathrm{x}}$. Therefore, even an infinite pseudomorphically strained superlattice thickness can be realized from a sequence of $G a A s_{1-x} N_{x}$ and $\ln A s_{1-y} N_{y}$ layers if the thickness of each layer is kept below the threshold for its lattice relaxation. Figure-1 shows the band edge variation (red curve) in 20 period of $\mathrm{GaAs}_{1-\mathrm{x}} \mathrm{N}_{\mathrm{x}} / \ln \mathrm{As} \mathrm{s}_{1-\mathrm{x}} \mathrm{N}_{\mathrm{x}}$ superlattice lattice matched to InP. As we have discussed earlier, the quaternary alloy GalnAsN could be a viable material for the application in IR devices, but as seen under the band 
anticrossing model approximation [13] the band gap of InGaAsN lattice matched on InP (blue curve) is much higher than that of the superlattice for the same nitrogen content. Hence the given superlattice band gap can be achieved for much lower nitrogen concentration in comparison to that in InGaAsN quaternary. This is of great technological advantage, due to the fact that the higher nitrogen concentration is hard to incorporate in the alloys and it also deteriorates the quality of the alloy.

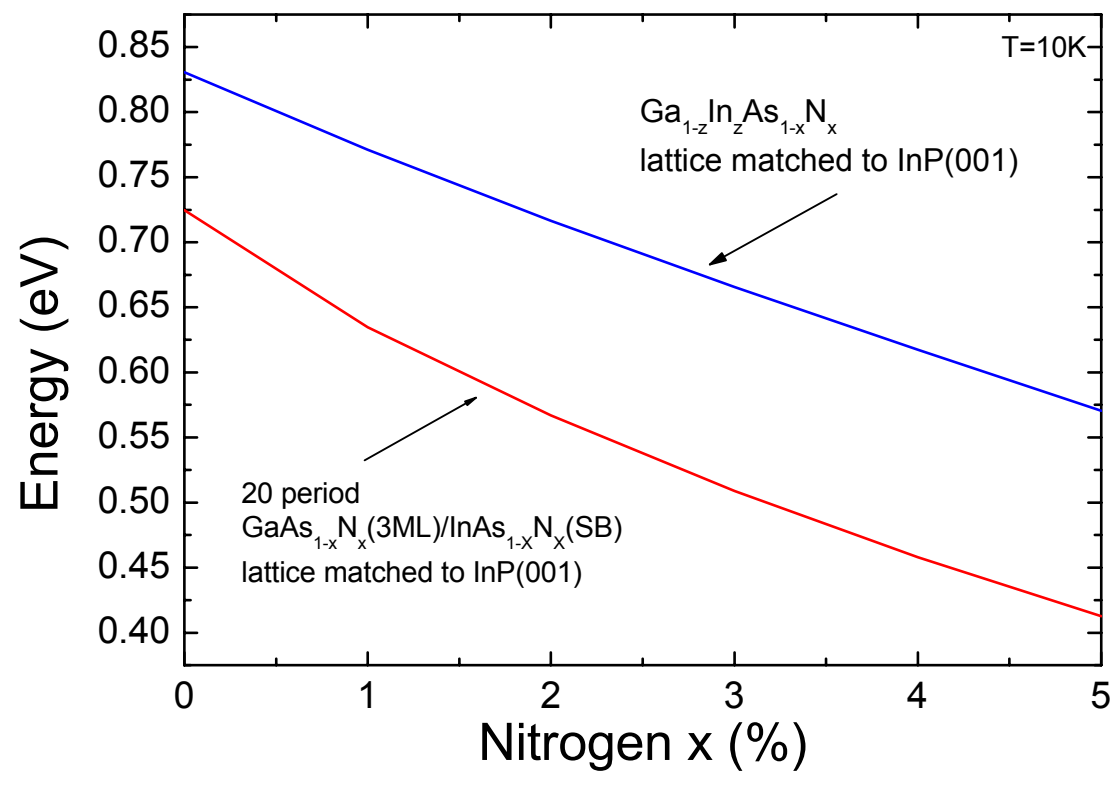

Figure 1. Evolution of the band edge energy gaps at $10 \mathrm{~K}$ in a 20 period $\mathrm{GaAs}_{1-\mathrm{x}} \mathrm{N}_{\mathrm{x}}(3 \mathrm{ML}) / \mathrm{InAs}_{1-\mathrm{x}} \mathrm{N}_{\mathrm{x}} \mathrm{SL}$ as a function of the nitrogen composition in the SL (red curve, after Ref.10). Note for each different nitrogen data point the thickness of the $\ln A s_{1-x} N_{x}$ sub-layers (4-9MLs) is adjusted to satisfy the lattice matching of the SL to InP (001). For comparison purpose band edge of quaternary GalnAsN is also shown (blue curve).

Figure $2 \mathrm{~b}$ ) shows the schematic of the insertion of the superlattice in the I region of the conventional $p$-I- $n$ diode shown in Figure 2a), with a schematic of the superlattice in figure $2 \mathrm{c}$ ). Also shown in figure $2 \mathrm{c}$ ) is the formation of miniband structures in conduction and valance bands, which determines the effective band gap of the structure. The effect of insertion of such a superlattice-like alloy within the intrinsic region of a $0.74 \mathrm{eV}$ InGaAs $p$-I-n diode was previously evaluated and it was shown that such a single junction device exhibits a photovoltaic response comparable to its lattice-mismatched $0.55 \mathrm{eV}$-InGaAs counterpart as shown in figure 3 [after Ref. 14]. In this work we have extended the approach to multi-junctions devices. Here three or more subcells with different effective bandgaps for the superlattice region are monolithically series connected. Maximum power output and performance of double, triple and quadruple junction TPV cells are evaluated as a function of the superlattice/cell design at a given black body emitter temperature. The study stresses the potential of the proposed approach for a significant enhancement of TPV converter performance.

Following the treatment of the Olson et.al. [15], we can make following simplifying assumption for the well behaved $p-I-n$ diode: 1) transparent zero resistance tunnel-junction interconnects, 2) no reflection losses, 3) no series resistance losses, 4) junctions collects every photon absorbed and 5) I-V curves are described by the ideal $(n=1)$ equation. The short circuit current density $\left(J_{s c}\right)$ of $i^{\text {th }}$ subcell is determined by the quantum efficiency of the subcell, $Q(\lambda)$ and by the spectrum of the light incident on the that cell, $\phi(\lambda)$ as, 
a)

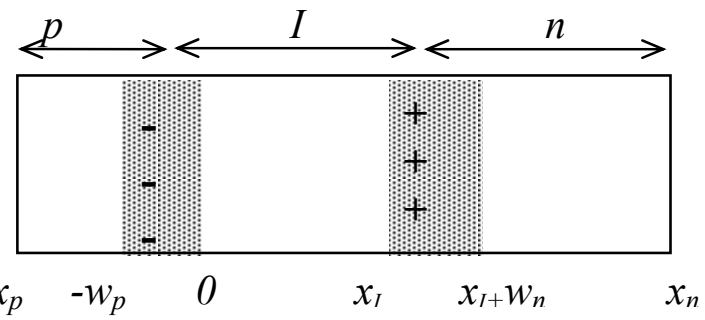

b)

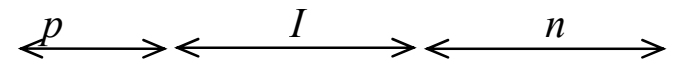

c)

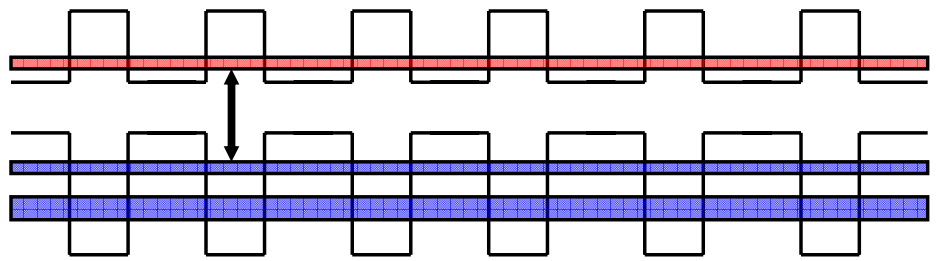

Figure2. Schematic of the $p-I-n$ diode, defining different parameters. a). Conventional $p-I-n$ device. b). $p-I-n$ device, with I-region filled with a GaAsN/InAsN superlattice. c) Schematic of the superlattice and the minibands. Vertical arrow in c) determines the distance between the miniband edges to give the effective band gap of the superlattice structure.

$J_{S C}^{i}=q \int_{0}^{\infty} Q_{i}(\lambda) \phi(\lambda) d \lambda$

Based on the assumptions we have made, quantum efficiency can be assumed simply dependant on the total thickness, $x$ of the device, as

$$
Q_{i}(\lambda)=1-\exp \left[-\alpha_{i}(\lambda) x_{i}\right]
$$

because the fraction $\exp [-\alpha(\lambda) x]$ of the incident light is transmitted through the cell instead of being absorbed. For the photons with wavelength greater than the band gap, $\alpha(\lambda)=0$, and hence $\exp [-\alpha(\lambda) x]=1$. The incident radiation flux $\phi(\lambda)$ on the top cell is simply the flux $\phi_{B B}(\lambda)$ of the black body radiation hitting the top surface of the cell. On the other hand, the photon flux hitting the bottom or lower cells is filtered by the top cells, so the bottom cell only sees an incident spectrum reduced by the factor of $\exp \left[-\alpha_{1}(\lambda) x_{1}\right]$ of $\phi_{B B}(\lambda)$, where $\alpha_{1}(\lambda)$ and $x_{1}$ are the absorption coefficient and the thickness of the top or the first cell. In general the short circuit current of $i^{\text {th }}$ cell in the multijunciton cell comprised of $m$ subcells can be given as,

$$
J_{S C}^{i}=q \int_{0}^{\lambda_{i}}\left(1-\exp \left[-\alpha_{i}(\lambda) x_{i}\right]\right) \exp \left[-\sum_{i=2}^{m} \alpha_{i-1}(\lambda) x_{i-1}\right] \phi_{B B}(\lambda) d \lambda
$$


For example, in three-cell system, the above relation for top $(i=1)$ and bottom ( $i=3)$ cell becomes,

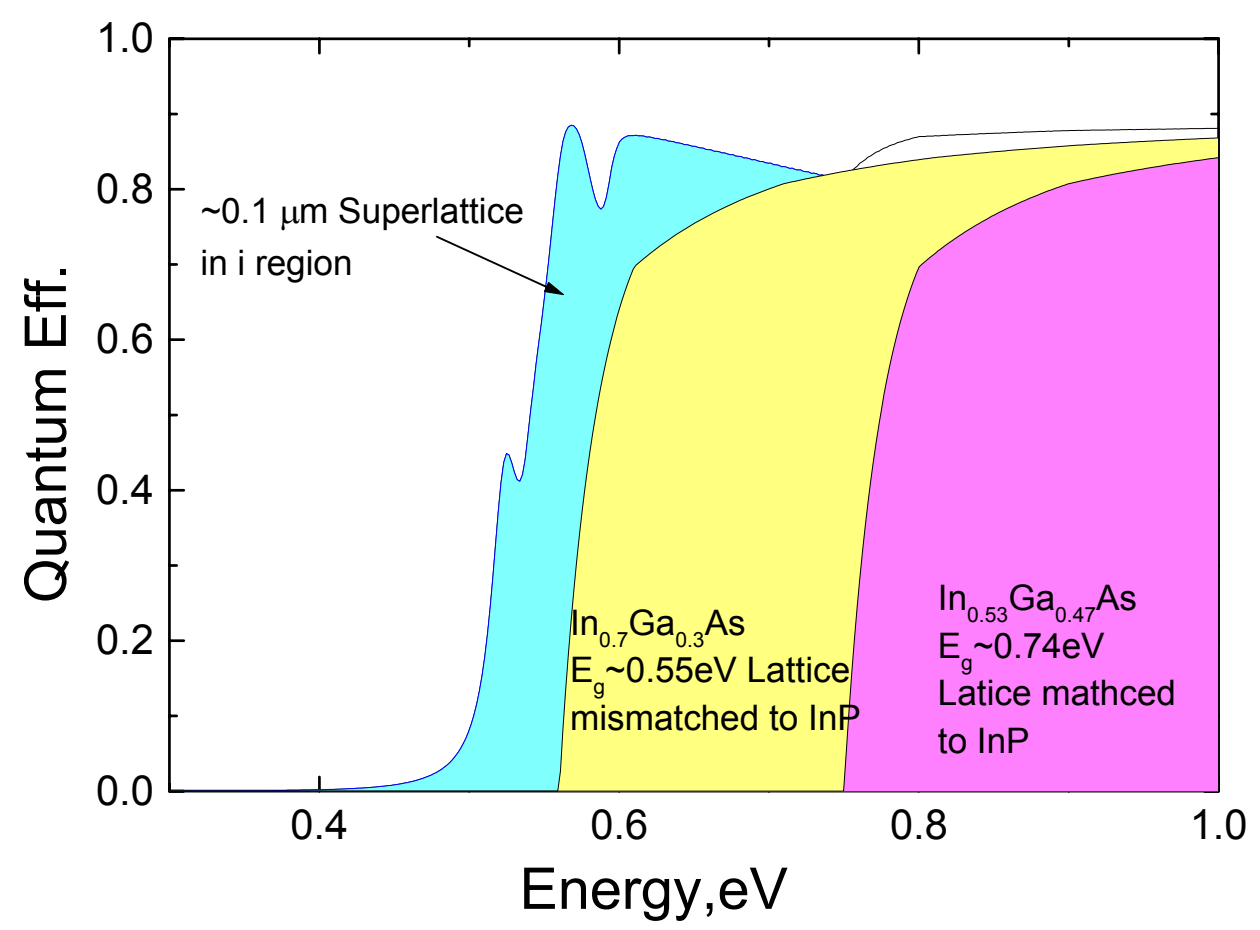

Figure 3. (After Ref. 14). Increased photocurrent response due to the addition of superlattice in $I$ region of $p-I-n$ device. For comparison purpose effects of InGaAs lattice matched and lattice mismatched to InP are also shown.

$J_{S C}^{1}=q \int_{0}^{\lambda_{1}}\left(1-\exp \left[-\alpha_{1}(\lambda) x_{1}\right]\right) \phi_{B B}(\lambda) d \lambda$

and

$J_{S C}^{3}=q \int_{0}^{\lambda_{3}}\left(1-\exp \left[-\alpha_{3}(\lambda) x_{3}\right]\right) \exp \left[-\left(\alpha_{1}(\lambda) x_{1}+\alpha_{2}(\lambda) x_{2}\right)\right] \phi_{B B}(\lambda) d \lambda$

respectively. We can assume that last cell is infinitely thick to absorb all the photons, giving, for example, $J_{S C}$ of last cell in three-junction cell as

$J_{S C}^{3}=q \int_{0}^{\lambda_{3}} \exp \left[-\left(\alpha_{1}(\lambda) x_{1}+\alpha_{2}(\lambda) x_{2}\right)\right] \phi_{B B}(\lambda) d \lambda$

Classical ideal photodiode $J-V$ equation can be written as, 
$J_{i}=J_{S C}^{i}-J_{0}\left[\exp \left(q V_{i} / k T\right)-1\right]$

where the dark current density $J_{0}$ is given as,

$J_{0}=J_{0, \text { base }}+J_{0, \text { intrinsic }}+J_{0, \text { emitter }}$

with different terms given as,

$$
\begin{aligned}
& J_{0, \text { emitter }}=q n_{I} \frac{D_{n}}{N_{A} L_{n}} \frac{\left\{\frac{S_{n} L_{n}}{D_{n}} \cosh \left(\frac{x_{p}-w_{p}}{L_{n}}\right)+\sinh \left(\frac{x_{p}-w_{p}}{L_{n}}\right)\right\}}{\left\{\frac{S_{n} L_{n}}{D_{n}} \sinh \left(\frac{x_{p}-w_{p}}{L_{n}}\right)+\cosh \left(\frac{\left.x_{p}-w_{p}\right)}{L_{n}}\right)\right\}} \\
& J_{0, \text { base }}=q n_{I} \frac{D_{p}}{N_{D} L_{p}} \frac{\left\{\frac{S_{p} L_{p}}{D_{p}} \cosh \left(\frac{x_{n}-\left(w_{p}+x_{I}\right)}{L_{p}}\right)+\sinh \left(\frac{x_{n}-\left(w_{p}+x_{I}\right)}{L_{p}}\right)\right\}}{\left\{\frac{S_{p} L_{p}}{D_{p}} \sinh \left(\frac{x_{n}-\left(w_{p}+x_{I}\right)}{L_{p}}\right)+\cosh \left(\frac{x_{n}-\left(w_{p}+x_{I}\right)}{L_{p}}\right)\right\}} \\
& J_{0, \text { int rinsic }}=q n_{I}^{2}\left(x_{I}+w_{p}+w_{n}\right) B_{\text {rad }}
\end{aligned}
$$

Here, $L$ is the diffusion length, $S$ is the surface recombination speed, $n_{l}$ is the intrinsic carrier concentration in the intrinsic region, $\mathrm{D}$ is the diffusion coefficient and the distance parameters $x$ and $w$ are defined in Fig. 2. Radiative recombination coefficient $B_{\text {rad }}$ is carrier density independent property of the material and is given as [16],

$B_{\text {rad }}=\frac{2 \pi n_{s}^{2}}{n_{I}^{2} h^{3} c^{2}} \int_{0}^{\infty} \alpha(E) e^{-E / k T} E^{2} d E$

where, $n_{s}$ is refractive index of the material, $h$ is the Planck's constant, $c$ is the speed of light. From equation-7, the voltage across the $i^{\text {th }}$ subcell is given as,

$V_{i}(J)=\frac{k T}{q} \ln \left(\frac{J_{S C}^{i}-J}{J_{0}^{i}}+1\right)$

and the voltage across the tandem device comprised of $m$ subcells can be given as,

$V_{\text {total }}(J)=\sum_{i=1}^{m} V_{i}(J)=\frac{k T}{q} \sum_{i=1}^{m} \ln \left(\frac{J_{S C}^{i}-J}{J_{0}^{i}}+1\right)$

Total power produced by this device can be given as, 


$$
P_{\text {total }}(J)=J \sum_{i=1}^{m} V_{i}(J)=J \frac{k T}{q} \sum_{i=1}^{m} \ln \left(\frac{J_{S C}^{i}-J}{J_{0}^{i}}+1\right)
$$

To find the current giving the optimal power, we can use the following condition,

$$
\frac{\partial P_{\text {total }}(J)}{\partial J}=0
$$

giving

$$
T(J)=\sum_{i=1}^{m}\left[\ln \left(\frac{J_{S C}^{i}-J}{J_{0}^{i}}+1\right)-\left(\frac{J}{j+J_{S C}^{i}+J_{0}^{i}}\right)\right]=0
$$

This transcendental equation has to be solved numerically to find the value of $J$ for a given stack of the tandem device. The value of $J$ found then can be used in equation 15 to find the optimal power of the system.

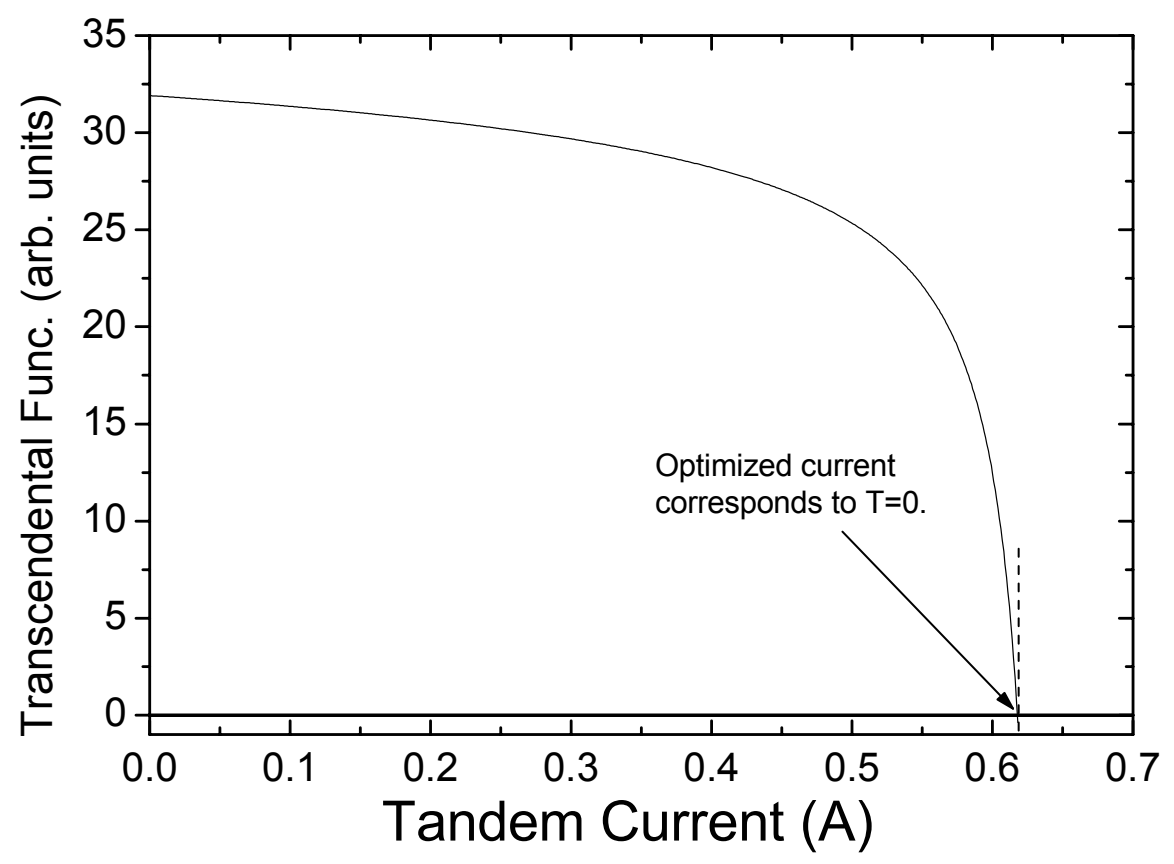

Figure 4. Transcendental expression of equation 17 plotted as function of current. Transcendental function of equation $=0$ determines the optimal current through the system.

Definition of the efficiency of the TPV system is generally very vague and depends on the device, radiation source, structure, converter and source separation and many other considerations $[17,18]$. One specific example of efficiency is radiative heat conversion efficiency as described by Mahorter et.al. [19]. In this study instead of efficiency we have focused on the power output density, an approach adopted by many authors, for example in ref. [20], to determine the device performance, since it depicts the performance of the converter itself, which is the 
main intention of this work. We consider the blackbody radiator operating at a temperature of $1350 \mathrm{~K}$ and the tandem temperature as $300 \mathrm{~K}$. In the first approach the band gap of first cell is fixed to $0.74 \mathrm{eV}$, which is the band gap of the InGaAs lattice matched to $\operatorname{InP}$ to find the optimized current through the system giving the maximum power output by varying the band gap of the second subcell. Current through the first cell is $0.64 \mathrm{~A} / \mathrm{cm}^{2}$ and by solving the transcendental equation 17, as shown in figure 4, optimal current for the two subcell tandem is found to be $0.618 \mathrm{~A} / \mathrm{cm}^{2}$ when the summation $T(J)$ goes to zero. Once the optimal current for the given set of tandem is found, equation-15 can be used to determine the output power of the tandem. For a fixed first subcell bandgap, second subcell bandgap can be varied to find the maximum power output of the tandem, giving the corresponding second subcell bandgap as the optimal band gap of the double junction tandem. Following the procedure, we can find the band gaps of the second $(0.625 \mathrm{eV})$, third $(0.535 \mathrm{eV})$ and fourth $(0.46 \mathrm{eV})$ subcells to achieve maximum power output in double, triple and quadruple junction cells respectively. Figure 5 shows the result of the procedure discussed above for the double, triple and quadruple junction devices, giving the output power density variation with the change of the band gap of the last subcell. Solid curves show the optimal powers for the subcell consisting bulk like lattice mismatched InGaAs in the conventional $p-I-n$ structure, on the other hand, the broken curves are the calculations using the superlattice in the I region of the $p-I-n$ structure. We can notice the better output performance using the superlattice, in addition to the lattice matching on the InP substrate. Blue and red dots respectively shows the results of the theoretical and experimental work performed by

Wanlass et al [20] and Wilt et al [21].

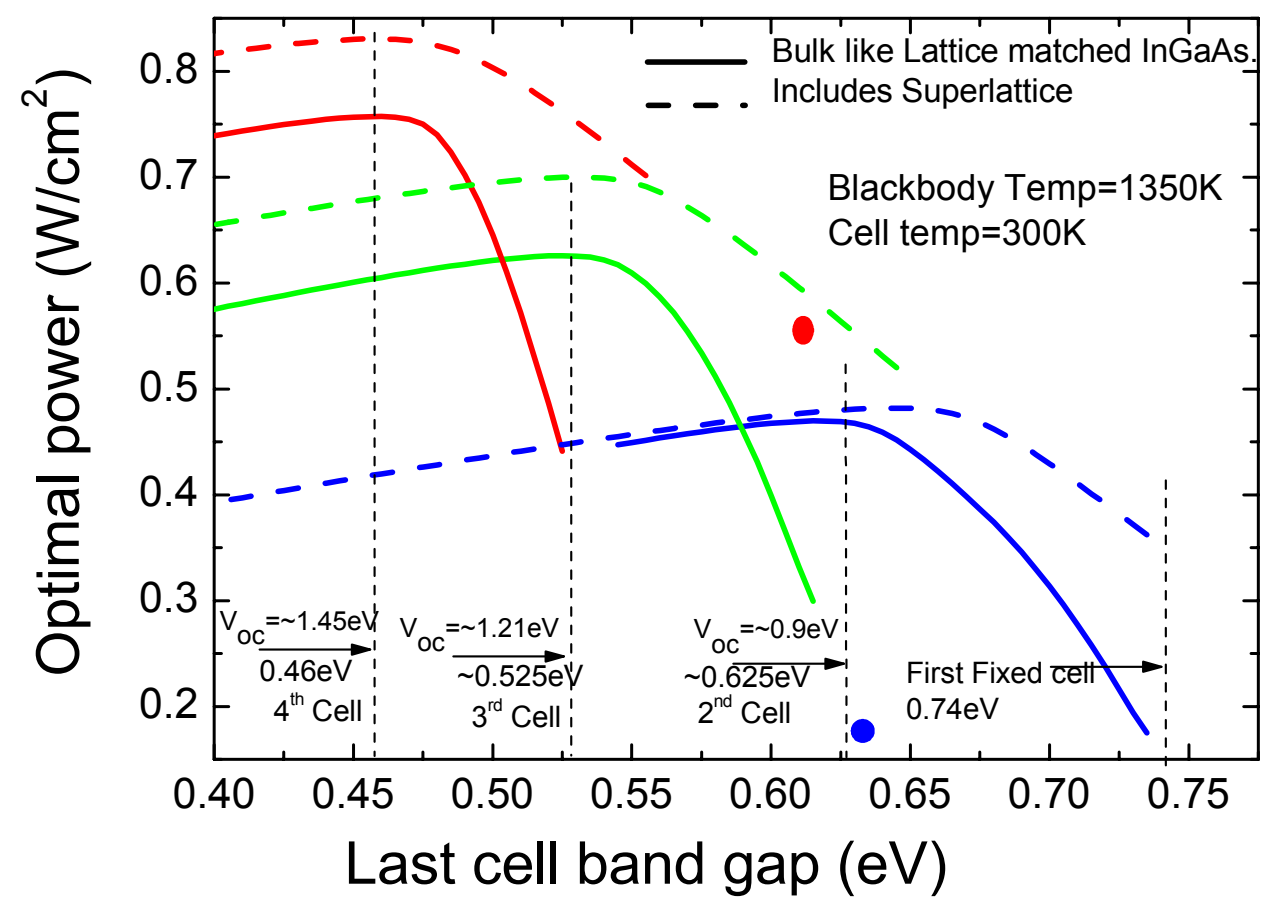

Figure 5. Power output for double (blue), triple (green) and quadruple (red) junction cells. Maximum power on each curve gives the optimal band gap for second $(0.615 \mathrm{eV})$, third $(0.525 \mathrm{eV})$ and fourth $(0.46 \mathrm{eV})$ subcells for fixed first $(0.74 \mathrm{eV})$ subcell. Solid curves are for the subcells with lattice mismatched InGaAs materials and the broken curves are calculated including the superlattice materials in the I region of the $p-I-n$ device.

If the first band gap is not fixed, optimal band gaps of the first two subcells also can be found by searching all the possible combination of the band gaps of the first subcell and the second subcell, giving the iso-power surfaces or iso-power curves as shown in figures 6 and 7 for the lattice mismatched InGaAs devices and in figure 8 for the superlattce device. It can be seen that the optimal band gaps for first and second subcells comes out to be at 
$0.64 \mathrm{eV} / 0.46 \mathrm{eV}$ for the lattice mismatched InGaAs devices and $0.62 \mathrm{eV} / 0.41 \mathrm{eV}$ for the superlattice devices respectively. It is important to note that the power output density of the tandem device made of superlattice (fig 8) is much higher than that of the lattice mismatched InGaAs (fig 7).

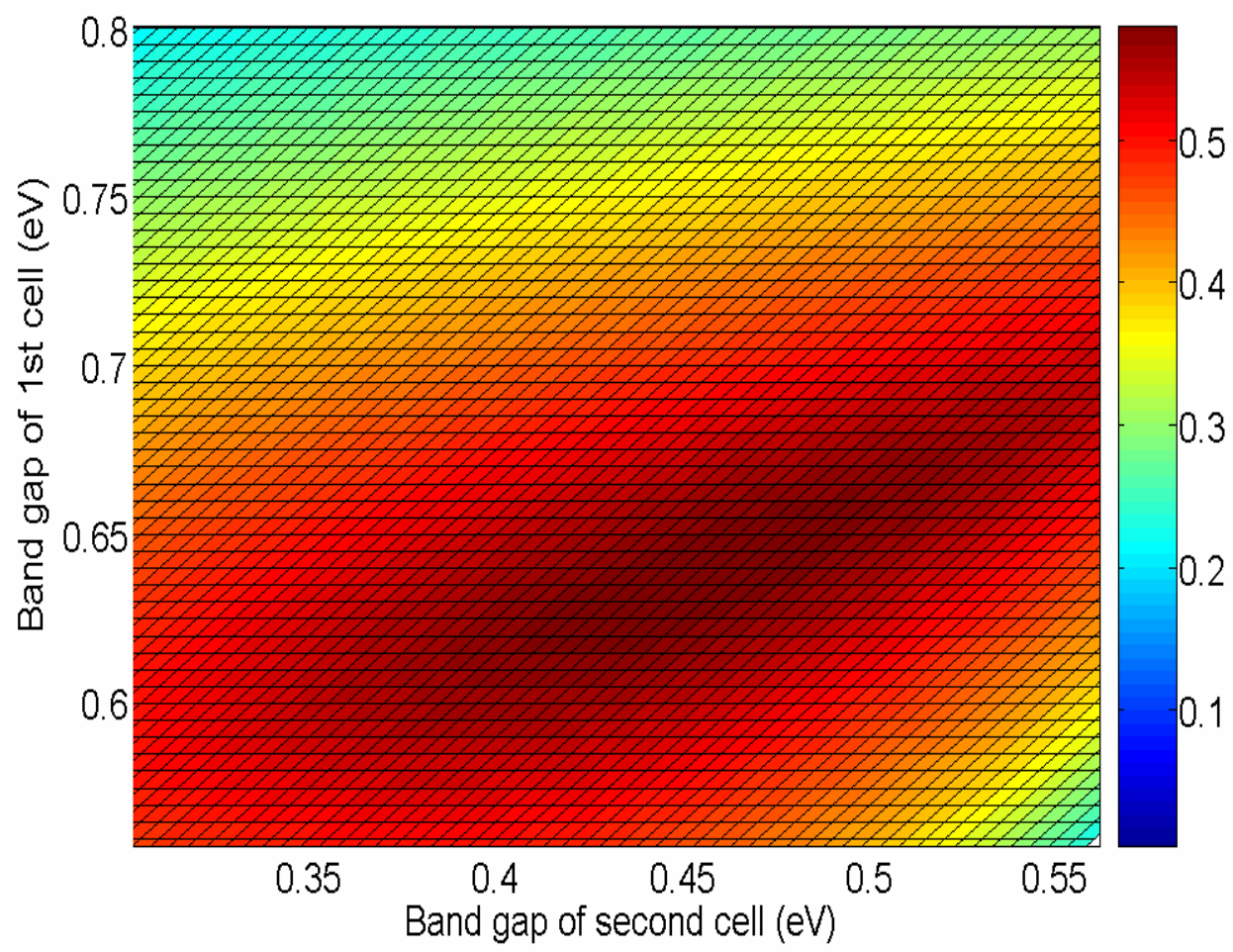

Figure 6, Iso-power surface for the band gap variation of top two subcells in double junction tandem consisting of lattice mismatched InGaAs. Colored scale shows the power in $\mathrm{W} / \mathrm{cm}^{2}$. It can be seen that maximum output power can be reached with slight variation for different band gap combinations.

As we have discussed that the lower band gaps needed for the subcells of multijunction device can be obtained from an lattice mismatched InGaAs layers on InP, with consequence of poor device performance due to the strain related problems. Replacing the I region with unstrained lattice matched GaAsN/InGaAs SL would avoid the presence of strain in the device on the one hand but on the other hand would reduce the active absorbing layer thickness, which is limited to only the I region of the subcell, since $p$ and $n$ region would be an unstrained InGaAs with fixed band gap of $0.74 \mathrm{eV}$. One consequence of thinner active absorbing region would be lesser current density and lesser power output. Since the absorption coefficient of the GaAsN/InAsN SL is higher than that of bulk InGaAs [14], I region filled with a superlattice will perform better than the I region of InGaAs, but since the overall active absorption region would be decreased, there will be a trade off between the strain effects on the device and the maximum power output. Since for the many applications, longevity of the device is an issue, it is important to have stable devices, even compromising the overall device output yield. With a use of relatively thicker superlattice we can gain the power density output equivalent or even better in comparison to the lattice mismatched devices. For a quadruple cell, the power density output reached more than $0.8 \mathrm{~W} / \mathrm{cm}^{2}$ for the superlattice thickness of $0.2 \mu \mathrm{m}$ for the second and third subcell and $\sim 1 \mu \mathrm{m}$ for the fourth subcell, which further can be increased by optimizing the thickness and other physical properties of the individual cell. Main reulsts of the paper can be summarized in the figure 9. This figure shows the variation of the output power density of the double, triple and quadruple tandem device with and without the use of a superlattice. The optimization of both the band gaps for double junction tandem with and without the superlatices are shown as green and black dots respectively. Hence higher output power can be reached using the optimized double band gaps with the use of the superlattice. 


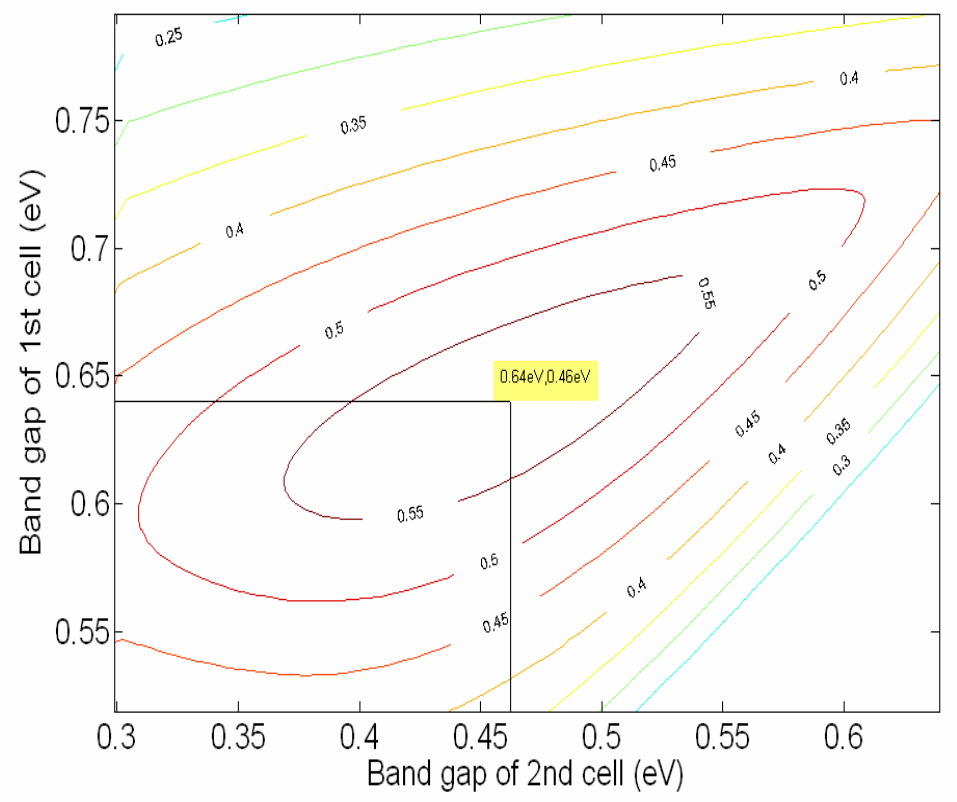

Figure 7. Iso-power curves representing combination of band gaps of first and second subcells made of lattice mismatched InGaAs to produce a given output power. Maximum power output can be reached for band gaps of 0.64 and $0.46 \mathrm{eV}$ for first and second subcells respectively.

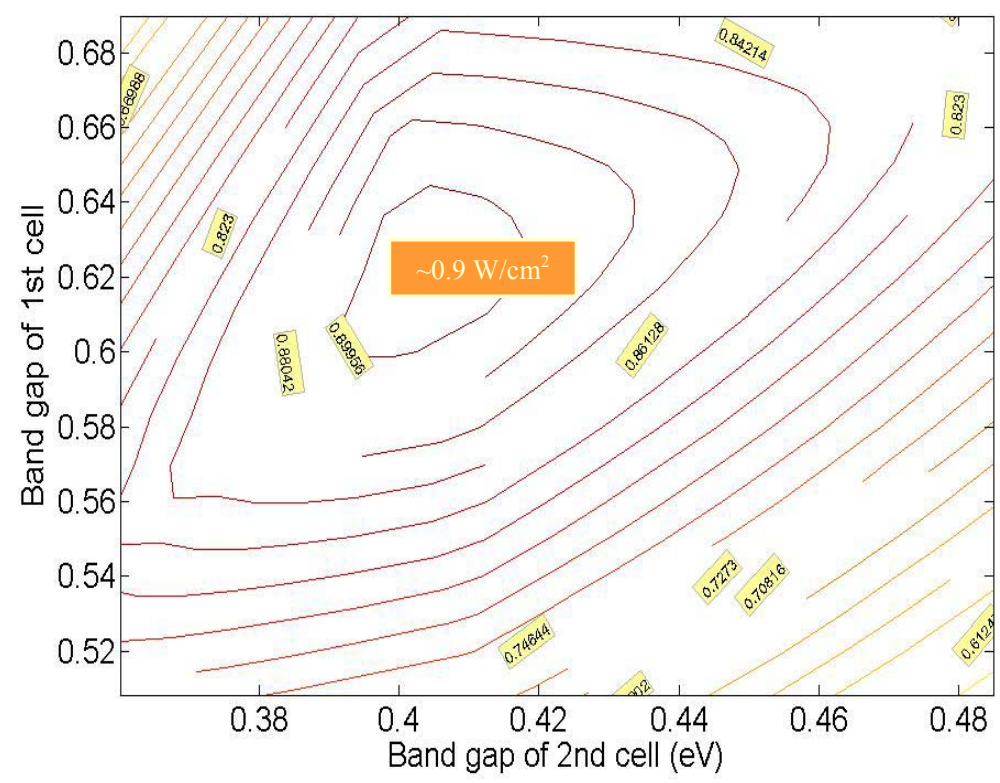

Figure 8. Iso-power curves representing combination of band gaps of first and second subcells made of superlatice to produce a given output power. Maximum power output can be reached for band gaps of $\sim 0.62$ and $0.41 \mathrm{eV}$ for first and second subcells respectively. 


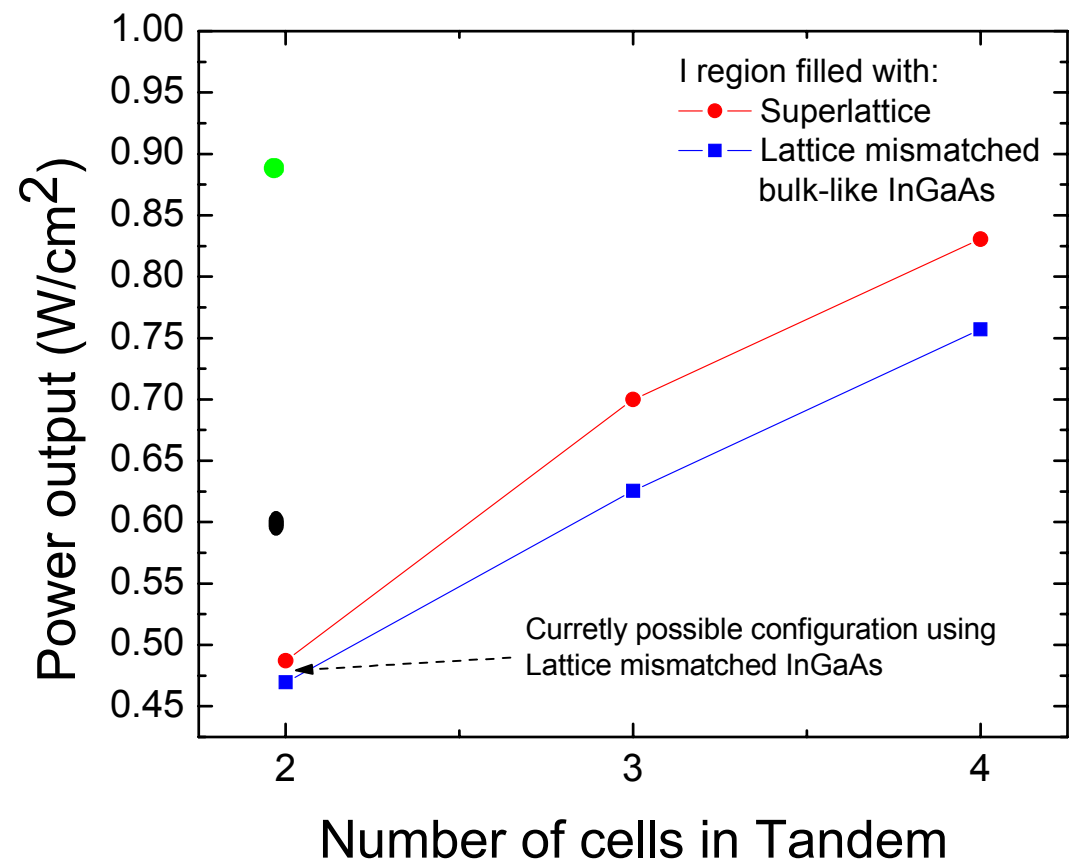

Figure 9. Output power density vs. the number of tandem cells for the devices with and without the use of lattice matched superlattice. Green and black dots respectively show the output power for the double junction tandem with and without the superlattice, in which both the band gaps were optimized for the highest power output.

In conclusion we have proposed the use of strain balanced superlattice structure in TPV device to achieve variant bandgaps between $0.4-0.7 \mathrm{eV}$ as needed for the type of radaitive source used. We have shown that better or comparable performance of device can be achieved by replacing the I region of the subcell by strain balanced GaAsN/InAsN superlattice lattice matched to InP. Due to the lattice matching structures, it is technologically feasible to implement three or four junction cell on InP based devices, which otherwise in the case of lattice mismatching devices is hard to implement due to the requirement of thick graded layer between the cells. We also deduced the optimized band gaps for two-cell tandem device to produce optimal power.

This work was partially supported by State of Texas Higher Education Coordinating Board Advanced Technology grants \\#003652-0126-2001 and \\#003652-0316-2001 and the NASA Grants \#NNC04GB53N.

[1] M. Weyers, M. Sato and H. Ando, "Red Shift of Photoluminescence and Absorption in Dilute GaAsN Alloy Layers", Jpn. J. Appl. Phys. 31, L853, 1992.

[2] J. N. Baillargeon, K. Y. Cheng, G. E. Hofler, P. J. Pearah, and K. C. Hsieh, "Luminescence quenching and the formation of the $\mathrm{GaP}_{1-x} \mathrm{~N}_{x}$ alloy in GaP with increasing nitrogen content", Appl. Phys. Lett. $602540,1992$.

[3] M. Kondow, K. Uomi, A. Niwa, T. Kitatani, S. Watahiki and Y. Yazawa, "GalnNAs: A Novel Material for Long Wavelength-Range Laser Diodes with Excellent High-Temperature Performance", Jpn. J. Appl. Phys. 35, $1273,1996$.

[4] S. R. Kurtz, A. A. Allerman, E. D. Jones, J. M. Gee, J. J. Banas, and B. E. Hammons, "InGaAsN solar cells with $1.0 \mathrm{eV}$ band gap, lattice matched to GaAs", Appl. Phys. Lett. 74, 729, 1999.

[5] A. Wagner, C. Ellmers, F. Hohnsdorf, J. Koch, C. Agert, S. Leu, M. Hofmann, W. Stolz, and W. W. Ruhle, "(Galn)(NAs)/GaAs vertical-cavity surface-emitting laser with ultrabroad temperature operation range", Appl. Phys. Lett. 76, 271, 2000. 
[6] E-M. Pavelescu, C. S. Peng, T. Jouhti, J. Konttinen, W. Li, M. Pessa, M. Dumitrescu and S. Spanulescu, "Effects of insertion of strain-mediating layers on luminescence properties of 1.3- $\mu \mathrm{m}$ GalnNAs/GaNAs/GaAs quantum-well structures", Appl. Phys. Lett. 80, 3054, 2002.

[7] W. Li, T. Jouhti, C. S. Peng, J. Konttinen, P. Laukkanen, E-M Pavelescu, M. Dumitrescu, and M. Pessa, "Lowthreshold-current 1.32- $\mu \mathrm{m}$ GalnNAs/GaAs single-quantum-well lasers grown by molecular-beam epitaxy", Appl. Phys. Lett. 79, 3386, 2001.

[8] K Kohler, J Wagner, P Ganser, D Serries, Geppert, M Maier and Kirste," The realization of long-wavelength $(\lambda$ $\leq 2.3 \mu \mathrm{m}) \mathrm{Ga}_{1-x} \mathrm{In}_{x} \mathrm{As}_{1-y} \mathrm{~N}_{y}$ quantum wells on InP by molecular-beam epitaxy". J of Phys.: Condens. Matter 16, S2995, 2004.

[9]. Buyanova, I.A., Chen, W.M., and Monemar, B., "Electronic Properties of Ga(In)NAs Alloys," MRS Internet J. Nitride Semicond. Res. 6 (2), 1, 2001.

[10] L. Bhusal, A. Alemu and A. Freundlich, "Short-period strain-balanced $G a A s_{1-x} N_{x} / \ln A s_{1-x} N_{x}$ superlattices lattice matched to $\operatorname{lnP}(001)$ : A material for 0.4-0.6 eV midinfrared applications", Phys. Rev. B 72, 073309, 2005.

[11] L. Bhusal, A. Alemu and A. Freundlich, "Band alignments and quantum confinement in (111) GaAsN/InAs strain-balanced nanostructures", Nanotechnology 15, S245, 2004.

[12] A. Freundlich, Final report, "State of Texas Advanced Research Grant", 03652-0099 1999, September. (2002).

[13] W. Shan, W. Walukiewicz, J. W. Ager III, E. E. Haller, J. F. Geisz, D. J. Friedman, J. M. Olson, and S. R. Kurtz, "Band Anticrossing in GalnNAs Alloys", Phys. Rev. Lett. 82, 1221 (1999).

[14] L. Bhusal, A. Alemu and A. Freundlich, "New material lattice matched to InP for $0.45-0.6$ eV photovoltaic applications." $31^{\text {st }}$ IEEE PVSC Proc., 133, 2005

[15] J.M. Olson, D.J. Friedman and S. Kurtz, Ch 9 in "Handbook of photovoltaic science and engineering", Eds. A Luque, S Hegedus, John Wiley and sons, Ltd, 2003.

[16] J. Nelson, in "The physics of Solar cells", Ch 4. Imperial College Press, 2003.

[17] L M Fraas, J E Avery, HX Huang and R U Martinelli, "Thermophotovoltaic furnace-generator for the home using low bandgap GaSb cells", Semicond. Sci. Technol. 18, S165, 2003.

[18] Keith Emery, "Characterizing thermophotovoltaic cells", Semicond. Sci. Technol. 18, S228, 2003.

[19] R.G. Mahorter, B.W. ernsman, R.M. Thomas and R.R. Siergiej, "Thermophotovoltaic system testing"Semicond. Sci. Technol. 18, S232, 2003.

[20] M.W. Wanlass and D.S. Albin, "A Rigorous analysis of series connected, multi-bandgap, tandem thermophotovoltaics (TPV) energy converters", Sixth conference on thermophotovoltaic generation of electricity, AIP Conference Proceedings, 738, 462, 2004.

[21] D.M. Wilt, R.J. Wehrer, W.F. Maurer, P.P Jenkins, B. Wernsman and R.W. Schultz, "Buffer layer effects on Tandem InGaAs devices", Sixth conference on thermophotovoltaic generation of electricity, AIP Conference Proceedings, 738, 453, 2004.

[22] B. Wernsman, R.R Siergiej, S.D. Link, R.G. Mahorter, M.N. Palmisiano, R.J. Wehrer, R.W. Schultz, G.P. Schmuck, R.L. Messham, S. Murray, C.S. Murray, F. Newman, D. Taylor, D.M. DePoy, T. Rahmlow, "Greater than $20 \%$ radiant heat conversion efficiency of a thermophotovoltaic radiator/module system using reflective spectral control", IEEE Transaction on Electron devices, 51, 512, 2004. 